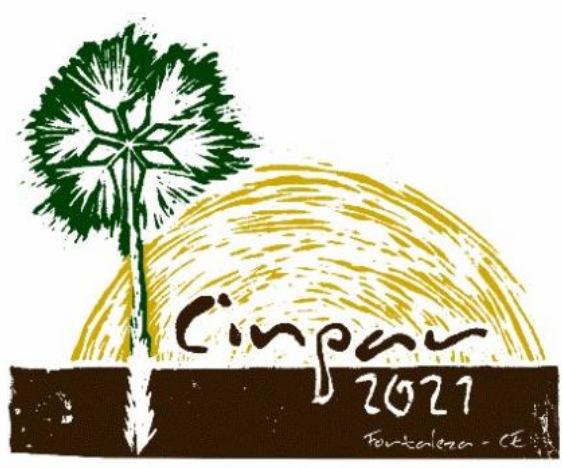

XVII Congresso Internacional sobre Patologia e Reabilitação das Construções

XVII Congreso Internacional sobre Patología y Rehabilitación de las Construcciones

XVII International Conference on Pathology and Constructions Rehabilitation

FORTALEZA (Brasil), 3 a 5 de junho de 2021

https://doi.org/10.4322/CINPAR.2021.163

\title{
Investigação de fissuras de fachadas com a termografia de infravermelho - estudos de laboratório
}

\section{Investigation of degree of damage of façade cracking with infrared thermography - laboratory studies}

\author{
Elton BAUER ${ }^{1}$, Patrícia M. MILHOMEM²
}

\author{
${ }^{1}$ Universidade de Brasília, Depto de Eng. Civil e Ambiental, Brasília/DF, Brasil,elbauerlem@gmail.com \\ ${ }^{2}$ Universidade de Brasília, Prog. Pós-Grad. Estrut. e Const. Civil, Brasília/DF, Brasil, patriciamotamilhomem@gmail.com
}

\begin{abstract}
Resumo: A termografia de infravermelho, aplicada como técnica de inspeção de fachadas no estudo de patologias, possui várias vantagens, onde se destaca: ser uma técnica não destrutiva, poder ser aplicada à distância, e fornecer os resultados imediatamente na forma de um termograma. Assim, é possível não só fazer o mapeamento de fissuras, mas também avaliar o grau de severidade dessas fissuras. Para isso se emprega a termografia quantitativa e se analisa os valores dos contrastes térmicos que indicam diferenças nque dependem da largura das fissuras. O objetivo deste estudo é observar em laboratório como evolui o contraste Delta $T$ para duas condições de fissuras, a saber larguras de $1,0 \mathrm{~mm}$ e $3,0 \mathrm{~mm}$. Para tal se desenvolveu um programa experimental de laboratório, em que em condições de aquecimento e arrefecimento se estudam os perfis de evolução do Delta $T$, associados as fissuras estudadas. Os resultados mostram perfis distintos do comportamento do contraste Delta T o que significa que é possível em campo identificar a severidade da degradação. Dessa forma se consegue quantificar a degradação nas fachadas de modo a auxiliar em estudos de patologias ou de vida útil.
\end{abstract}

Palavras-chave: fachada; fissuras; termografia de infravermelho; grau de dano.

\begin{abstract}
Infrared thermography applied as a facade inspection technique in the study of pathologies has several advantages, which stand out as being a non-destructive technique, being able to be applied at a distance, and providing the results immediately in the form of a thermogram. Thus, it is possible not only to map cracks, but also to assess the degree of severity of these cracks. For that, quantitative thermography is used and the values of the contrasts that indicate differences in the crack regions are analyzed. The purpose of this study is to observe in the laboratory how the Delta T contrast evolves for two crack conditions, namely widths of $1.0 \mathrm{~mm}$ and $3.0 \mathrm{~mm}$. To this end, an experimental laboratory program was developed, in which under heating and cooling conditions, the evolution profiles of Delta $T$ are studied, associated with the studied cracks. The results show different profiles of the Delta T contrast behavior, which means that it is possible in the field to identify the severity of degradation. In this way it is possible to quantify the degradation on the façades in order to assist in studies of pathologies or service life and degradation.
\end{abstract}

Key Words: facade, cracks, infrared thermography, damage. 


\section{Introdução}

A fissuração é uma das principais patologias observadas nas fachadas dos edifícios. Geralmente, se observa grande incidência da mesma em fachadas expostas a grande incidência da radiação solar, bem como também em zonas do topo do edifício (Souza e Bauer, 2018). A degradação por fissuração afeta a integridade da fachada o que leva a possíveis riscos estruturais. Por outro lado, uma vez que ocorram fissuras, outros agentes de degradação podem atuar mais incisivamente, como é o caso da chuva dirigida que pode infiltrar pelas fissuras. Sob outro enfoque, o aumento da fissuração é observado com o aumento da idade dos edifícios (Souza et al, 2018; Silvestre e Brito, 2018; Bauer et al., 2015). Essa tendência de comportamento tem como uma das principais causas o efeito cumulativo das ações climáticas sobre a fachada com o aumento associadoa da degradação (Bauer et al., 2016).

As inspeções de fachada são inseridas em processos de manutenção, de forma preventiva conforme define o plano de manutenção do edifício, e de forma preditiva quando é necessária a correção de alguma patologia observada (Flores-Collen et al.,2011). A inspeção de fachadas permite também o mapeamento de danos, e a partir das quantificações decorrentes é possível estudar a degradação e a vida útil (Nascimento et al., 2016). A execução da inspeção geralmente é uma atividade de logística complexa. Na maioria das vezes efetuada com o edifício em uso, sem interrupção das atividades de seus usuários.

A termografia de infravermelho como técnica de inspeção apresenta várias vantagens: não é destrutiva, pode ser feita a distâncias razoáveis (mais de $20 \mathrm{~m}$ ), e fornece o resultado de forma imediata através de um termograma (Bauer et al.,2016). Dado ao fato de que as patologias de fachada alteram localmente o fluxo de calor, é possível identificar, regiões mais quentes ou mais frias nos termogramas. Esse contraste é utilizado tanto para identificar a região com defeito, como também de forma quantitativa estabelecer associações com o grau de dano (Bauer et al.,2016). A técnica de inspeção usualmente empregada é a termografia passiva, onde se utiliza as variações de fluxo de calor originadas pelo meio ambiente (Maldague, 2001). Nesse caso, o entendimento do comportamento térmico da fachada, bem como das características do clima, em particular a incidência solar, são decisivos nas definições da inspeção (Maldague, 2001).

Se de uma forma a inspeção termográfica é vantajosa, de outra existem vários pontos críticos que devem ser mencionados. As condições de fluxo de calor sobre a fachada são dinâmicas ao longo do dia, o que faz com que os contrastes de temperatura, que identificam as patologias, se alterem de forma significativa. Isso significa que existem frequentemente situações em que é impossível mapear as patologias, principalmente quando o fluxo de calor é muito baixo (Freitas et al., 2014; Bauer et al. 2015). Sob outro enfoque, quando se busca quantificar o grau de dano de uma patologia, é necessário trabalhar com a termografia quantitativa. É necessário mensurar as temperaturas (região com defeito e sem defeito) de modo a determinar a severidade de uma patologia. Assim é necessário empregar avaliações precisas de parâmetros como: emissividade, temperatura aparente refletida, distância, umidade relativa, dentre outros. Nessa abordagem são estudadas evoluções e diferenças relativas entre as temperaturas obtidas dos termogramas. Maldague (2001) cita quatro funções de contraste principais, dentre elas: contraste absoluto, contraste corrido, contraste normalizado e contraste padrão. O contraste mais empregado é o absoluto conhecido também como DeltaT (Bauer et al. 2016, Freitas et al., 2014), que é utilizado no presente estudo.

Para a investigação termográfica da fissuração, com finalidade de estudar o grau de dano, é necessário o entendimento de diferentes comportamentos associados ao fluxo térmico. Em termos gerais se observa que no início do aquecimento as fissuras são regiões mais frias. Já quando ocorre inversão do fluxo térmico (após cessar a incidência do sol) as fissuras são caracterizadas como regiões mais quentes (Bauer et al., 2016, Bauer et al., 2018). Nessa mesma abordagem, a quantificação do Delta-T é diferenciada em função de características das fissuras (profundidade, largura). Existem questões importantes ainda não resolvidas, que dizem respeito a entender qual o padrão de evolução das temperaturas e do contraste, nas etapas de aquecimento e arrefecimento. Com base nesses padrões podem ser definidos os momentos ideais para se fazer as inspeções (maiores contrastes) e identificados comportamentos e valores que se associam ao grau de dano, além de obviamente, efetuar o mapeamento das fissuras. Este estudo se propõe a investigar o comportamento termográfico (em laboratório) de fissuras com larguras definidas, em ciclos de aquecimento e de arrefecimento de modo a contribuir no entendimento dos fenômenos envolvidos. 


\section{Materiais e métodos}

O programa experimental utiliza um conjunto grande de definições que envolvem a definição e construção dos corpos-de-prova, o método controlado de aquecimento e arrefecimento e a definição de critérios para obtenção das temperaturas e contrastes nos termogramas. O estudo se foca na investigação do comportamento de fissuras lineares de largura 1,0 e 3,0 mm, na profundidade de $10,0 \mathrm{~mm}$. As variáveis independentes são as larguras de fissuras e as variáveis dependentes são: temperaturas ao longo do tempo, Delta-T ao longo do tempo, ambos na etapa de aquecimento e arrefecimento. A Tabela 1 mostra a identificação das fissuras estudadas.

Tabela 1 - Características das fissuras estudadas

\begin{tabular}{|c|c|c|c|}
\hline Identificação & Largura $\mathbf{( m m})$ & Profundidade $\mathbf{( m m})$ & Tipo \\
\hline A & 1,0 & 10,0 & Linear \\
\hline B & 3,0 & 10,0 & Linear \\
\hline
\end{tabular}

\subsection{Definições dos corpos-de-prova}

Os corpos-de-prova são placas de argamassa nas dimensões de $180 \times 400 \mathrm{~mm}$, com espessura de $50 \mathrm{~mm}$, conforme ilustra a Figura 1. Todo o perímetro da placa é revestido com isolante térmico na espessura de 35 $\mathrm{mm}$. Esse cuidado é para que o fluxo térmico predominante seja no sentido da profundidade da placa. As placas são previamente fissuradas de modo a se obter fissuras lineares com a profundidade de $10 \mathrm{~mm}$ e largura de $1 \mathrm{~mm}$ (Placa A) e $3 \mathrm{~mm}$ (Placa B).

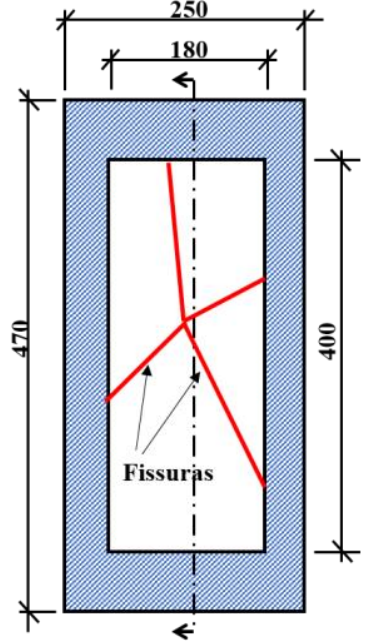

(a)
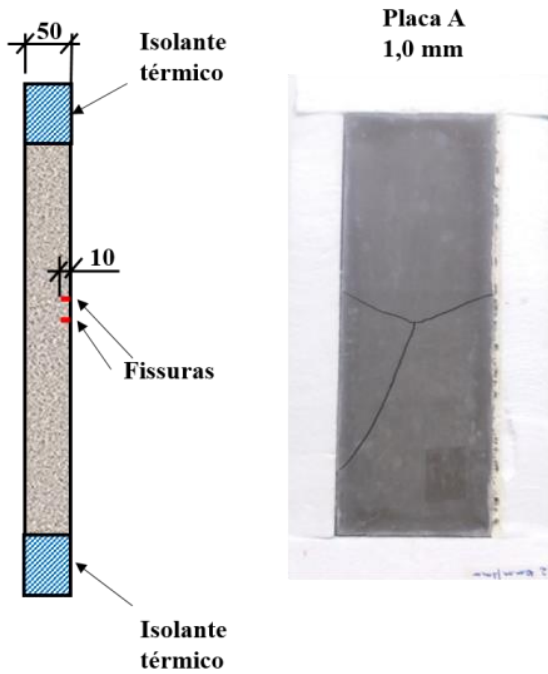

(b)

Figura 1 - (a) Detalhes dos corpos-de-prova; (b) Fotografias das placas A $(1,0 \mathrm{~mm})$ e B $(3,0 \mathrm{~mm})$

\subsection{Método de aquecimento e arrefecimento}

$\mathrm{O}$ aquecimento das placas é promovido por um dispositivo de lâmpadas infravermelho, divididas em dois conjuntos dispostos a esquerda e direita da placa de estudo (Figura 2). A potência ajustada é de $700 \mathrm{~W}$. A distância entre as lâmpadas e a placa é de $90 \mathrm{~cm}$. A câmara termográfica se posiciona ao centro, a uma distância de $230 \mathrm{~cm}$ das placas, de modo que sejam eliminados quaisquer problemas de reflexão oriunda do dispositivo de aquecimento.

O período de aquecimento definido é de 120 minutos, após o qual as lâmpadas são imediatamente desligadas se iniciando então o arrefecimento. Todo o ciclo tem uma duração de 240 minutos. As temperaturas máximas observadas são da ordem de 55 a $60^{\circ} \mathrm{C}$. Para cada análise é feita uma repetição do experimento. 


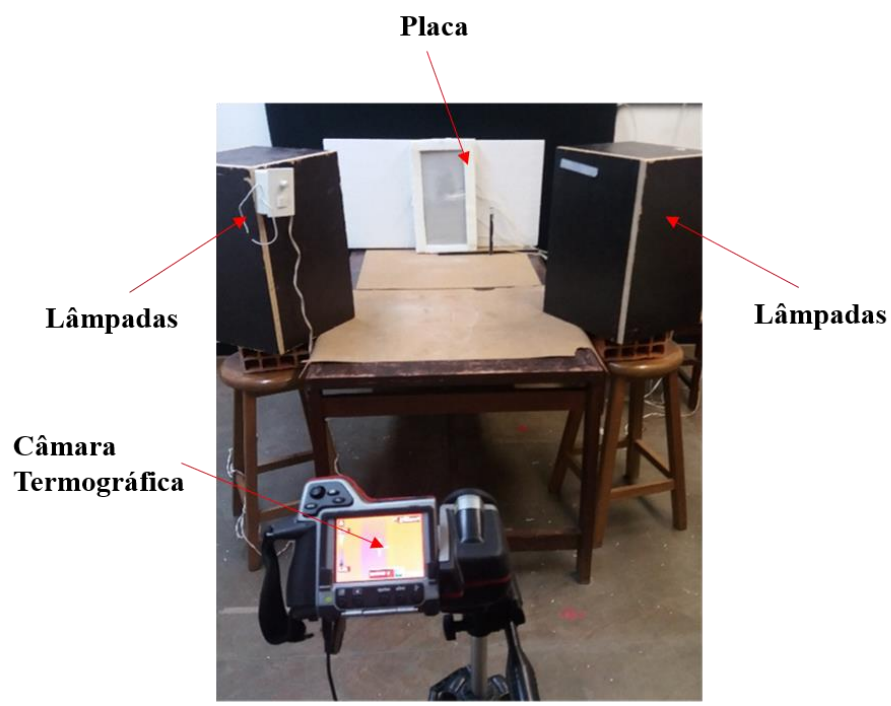

Figura 2 - Dispositivo de ensaio para o aquecimento e arrefecimento

\subsection{Critérios e definições para as avaliações termográficas}

As definições dos parâmetros de referência para análise termográfica quantitativa foram determinados preliminarmente em um ciclo de aquecimento e arrefecimento. A temperatura aparente refletida foi determinada de acordo com a norma ASTM E 1862-97. Para a fase de aquecimento o valor determinado é de $32,7^{\circ} \mathrm{C}$, e para o arrefecimento de $27,6^{\circ} \mathrm{C}$. A emissividade foi determinada no método da fita padrão de acordo com a Norma ASTM 1933-99. O valor determinado corresponde a 0,89.

A câmara termográfica é uma Flir T-400 com lente telescópica de $15^{\circ}$ acoplada. A especificação e os ajustes a utilizar são: faixa de temperatura -20 a $120^{\circ} \mathrm{C}$, faixa espectral 7,5 a $13,0 \mu \mathrm{m}$, resolução $320 \times 240$ pixels, IFOV $0,820 \mathrm{mrad}$. A obtenção dos termogramas é definida para uma frequência de 1 imagem a cada 4 minutos, ao longo dos 240 minutos do ciclo.

$\mathrm{Na}$ análise dos termogramas é apresentado o contraste absoluto (Delta T). O mesmo é calculado pela equação (1) a seguir.

$$
\text { Delta } T(\mathrm{t})=T d(t)-\operatorname{Tnd}(t)
$$

Onde: Delta $\mathrm{T}(\mathrm{t})$ é a diferença de temperatura (contraste) no tempo $\mathrm{t}, \mathrm{Td}(\mathrm{t})$ é a temperatura da área com defeito (fissura) no tempo $t$; Tnd(t) é a temperatura da área sem defeito no tempo $t$.

Para obter Td e Tnd se emprega o software Quick Report v 1.2. A cada pixel do termograma obtem-se um valor de temperatura. É possível exportar para uma planilha os valores de temperatura. O presente estudo utiliza a obtenção dos valores por meio de linhas de referência, conforme ilustra a Figura 3. No termograma são traçadas 3 linhas paralelas à fissura de cada lado, e exportadas para a planilha (Figura 3-a). Na análise dessas linhas, considera-se como espúrias as linhas cujos valores de temperatura medidos se afastam de mais de um desvio padrão da média dos valores. Das linhas restantes se calcula a nova média que corresponde ao valor de Tnd. Para determinação de Td, é traçada uma linha transversal à fissura (Figura 3-a) e exportada para a planilha. A análise dessa linha mostra um comportamento similar à Figura 3-b para a etapa de arrefecimento. Neste caso toma-se o valor da temperatura do pixel mais aquecido a qual corresponde à Td. Raciocínio inverso ocorre quando se tem o regime de aquecimento onde se toma o valor de temperatura do pixel de menor valor. 


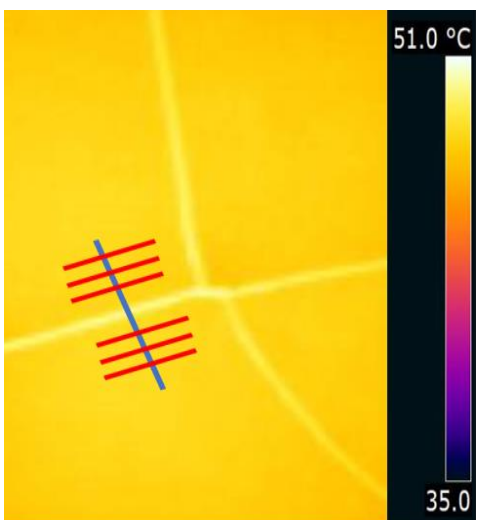

Determinação da Td

Determinação da Tnd

(a)

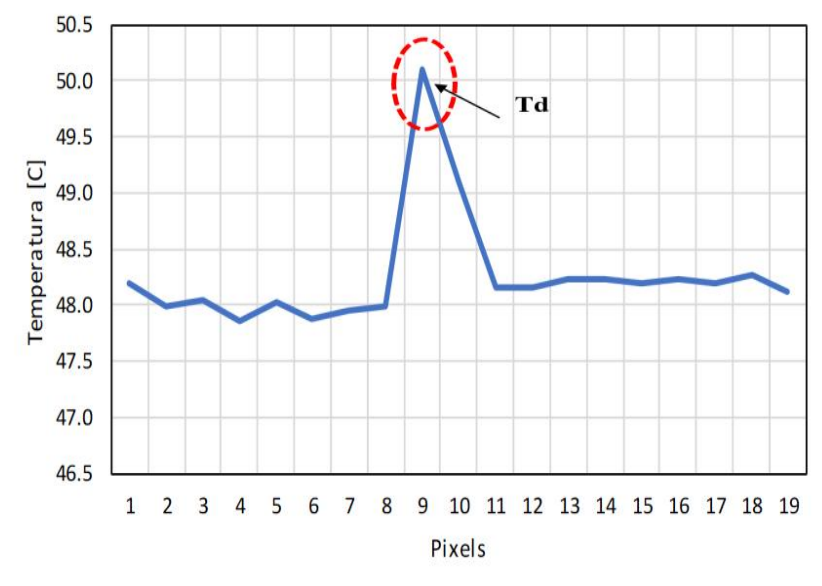

(b)

Figura 3 - (a) Termograma ilustrando as retas de determinação de Td e Tnd; (b) Perfil de temperatura ao longo dos pixels na reta transversa à fissura para determinação de Td (arrefecimento)

\section{Resultados}

A observação do comportamento capturado pelos termogramas é mostrada nas Figuras 4 e 5 . Para ambas as fissuras ( $A$ e B), no início do aquecimento as fissuras aparecem mais frias do que as regiões próximas sem defeito (Figura 4-a e Figura 5-a). Com a evolução do ciclo, o comportamento se caracteriza, ainda na etapa de aquecimento, por um aumento das temperaturas, e uma menor visualização das fissuras, que passam a apresentar temperaturas mais próximas da temperatura da região sem defeitos. Na Figura 4-b se observa que a fissura já é pouco visível aos 68 minutos (Fissura A). No mesmo tempo, a Fissura B (Figura 5-b) já mostra outra condição, em que a fissura é perfeitamente identificada no termograma. Com a evolução do aquecimento, e também com a transição do ciclo de aquecimento para arrefecimento, haverá um momento em que o fluxo térmico será nulo, e nessa condição a Td será igual a Tnd, sem visualiação da fissura.

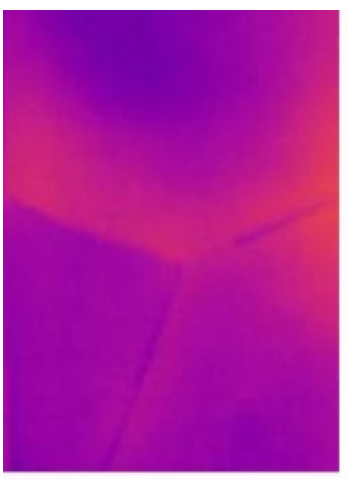

$12 \min$

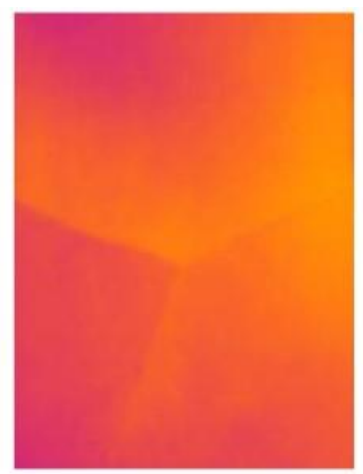

$68 \mathrm{~min}$

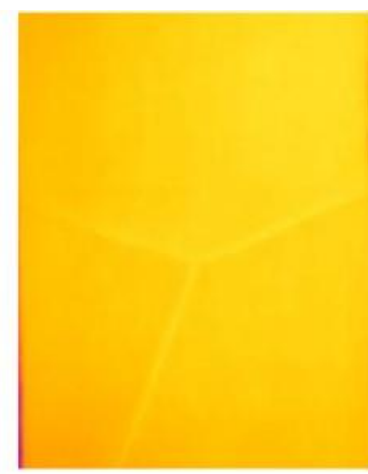

$132 \mathrm{~min}$

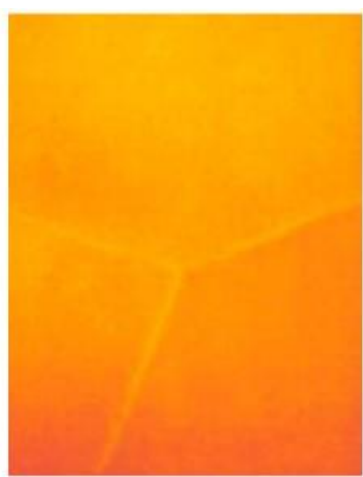

$204 \mathrm{~min}$

(a)

(c)

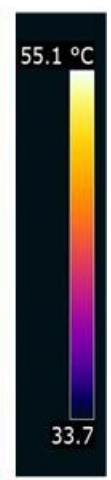

(d)

Figura 4 - Termogramas ilustrando o comportamento no aquecimento e arrefecimento para a fissura A.

Para o arrefecimento se idenfifica uma tendência similar para ambas as fissuras, ou seja, de apresentar Td superior a Tnd. Isso é ilustrado nas Figuras 4-c e 4-d, e também 5-c e 5-d. 


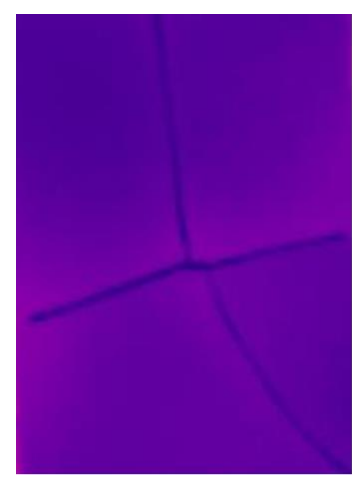

$12 \min$

(a)

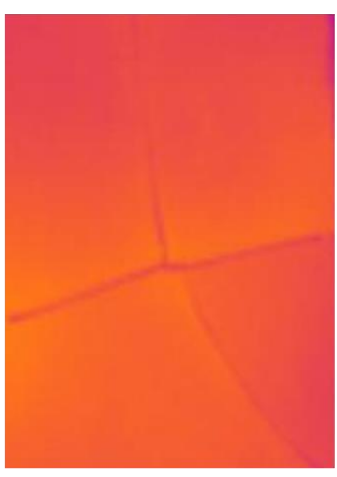

$68 \min$

(b)

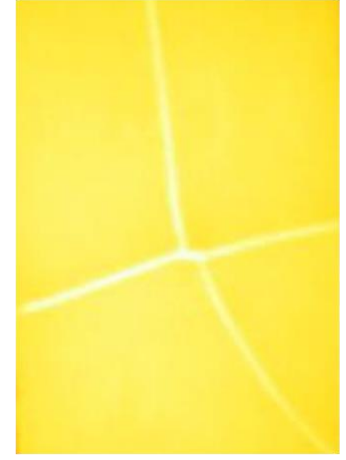

$132 \min$

(c)
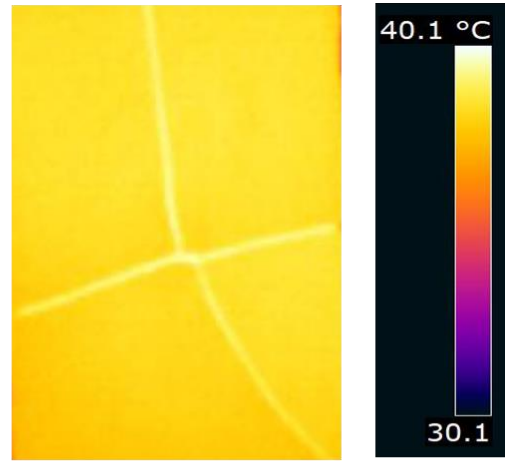

$204 \min$

(d)

Figura 5 - Termogramas ilustrando o comportamento no aquecimento e arrefecimento para a fissura B.

A análise da evolução das temperaturas (Td e Tnd) para cada situação ( $A$ e B) é necessária para entendimento dos comportamentos em estudo. A Figura 6-a apresenta a evolução das temperaturas para a Td, enquanto que a Figura 6-b traz a evolução para Tnd. Se constata que ambas as fissuras partem da mesma temperatura, correspondente a $25^{\circ} \mathrm{C}$, que é a condição de repouso do sistema. Na fase inicial se evidencia uma evolução do aquecimento como resposta à energia fornecida pelas lâmpadas. Se observa que a Fissura $A(1,0 \mathrm{~mm})$ tem um perfil de aquecimento em que tanto $\mathrm{Td}$ como Tnd, apresentam temperaturas superiores à Fissura $B(3,0$ $\mathrm{mm})$. A largura da fissura parece diferenciar o comportamento encontrado. A fissura $B$, por possuir abertura muito maior que a $\mathrm{A}$, permite trocas de convecção localizadas que geram temperatura inferiores comparadas à Fissura $A$, de menor abertura. Essa situação influencia também a Tnd, uma vez que essas temperaturas são obtidas em regiões próximas as fissuras (Figura 3-a).

Para a condição de arrefecimento (após 120 min.), o comportamento se inverte, ou seja, para a fissura B os valores de Tnd e Td são superiores. Nessa condição, o fluxo de calor ocorre do interior da placa para as superfícies em todas as direções. O termograma fornece as temperaturas observadas na face frontal da placa, onde estão as fissuras. Para esse caso, a fissura B, de maior abertura gera uma maior resistência térmica ao fluxo transversal de calor (no plano da face). Disso decorre que as temperaturas medidas são superiores na fissura (Td) e na região vizinha a mesma (Tnd). Maldague (2001) alerta para temperaturas superiores oriundas dessa condição.

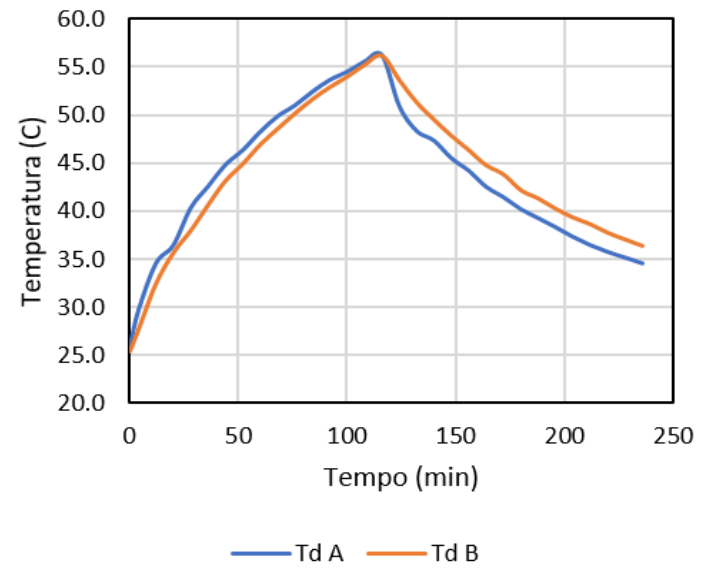

(a)

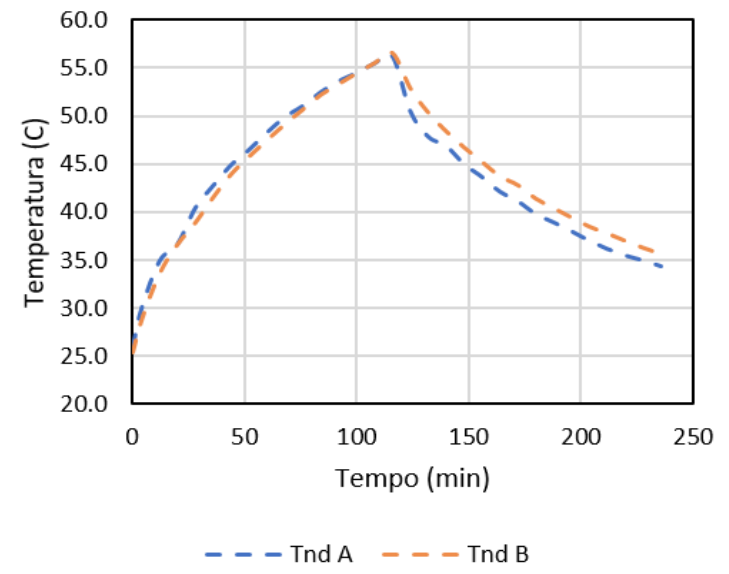

(b)

Figura 6 - (a) Evolução da temperatura na fissura (Td); (b) Evolução da temperatura na região sem defeito (Tnd).

O contraste Delta T obtido a partir dos termogramas é apresentado na Figura 7. No aquecimento o contraste é maior nos momentos iniciais conforme observado nas Figuras 4 e 5 . Com o crescimento das temperaturas o Delta T diminui. Esse fato é decorrente do aquecimento geral do sistema, onde as temperaturas aumentam no sentido da profundidade da fissura. Esse ponto é importante pois no caso das inspeções de campo a 
diminuição do Delta T implica em dificuldades para identificação das anomalias. Assim as condições de início ou inversão de fluxo são as mais propícias para a vistoria por apresentarem maior Delta T

A fissura $A$ evolui de forma decrescente nos valores de Delta $T$ no aquecimento, sendo que o mesmo se torna nulo aos 98 minutos. A fissura B apresenta valores de Delta $\mathrm{T}$ bem mais altos (em módulo) em todo o aquecimento. Esse comportamento permite definir um critério para o grau de dano atribuído à fissuração. Fissuras com maior abertura apresentam Delta T superior no aquecimento. No arrefecimento a fissura A possui maiores valores de Delta T do que na etapa anterior de aquecimento, sendo, entretanto, inferiores ao da Fissura B. Com a evolução do arrefecimento os valores de Delta T tendem a diminuir até a condição de equilíbrio ao final do ensaio. Como critério para as inspeções, se pode observar que ambas as fissuras são identificáveis com valores distintos de Delta T no período de arrefecimento. Assim se pode afirmar que no arrefecimento se tem condições mais adequadas para estudar essas duas fissuras.

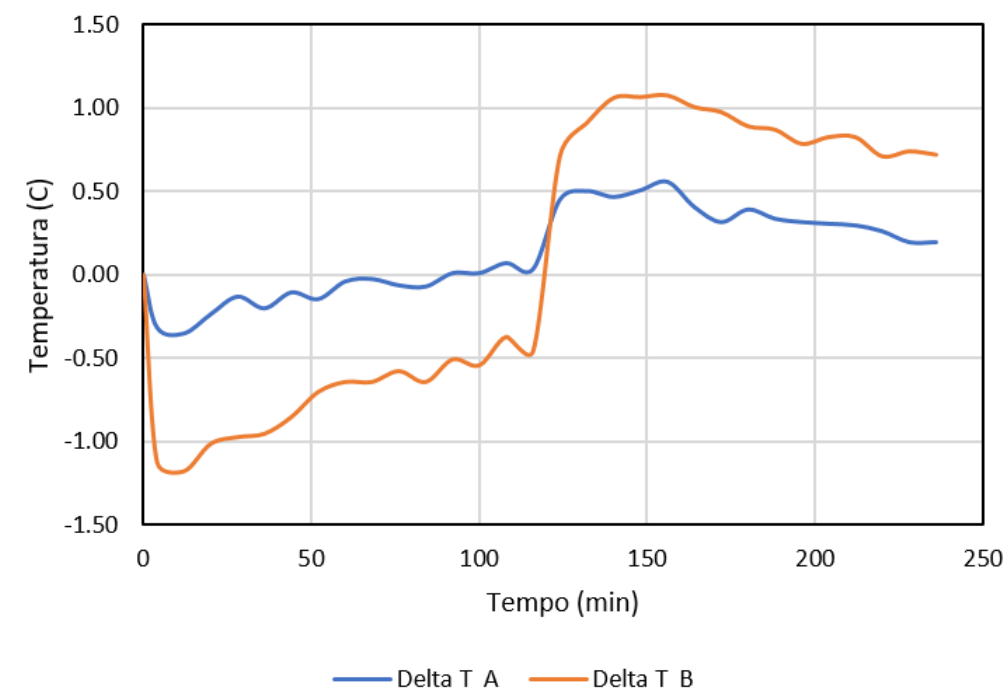

Figura 7 - Delta T nas etapas de aquecimento e arrefecimento para as fissuras A e B.

\section{Conclusões}

A investigação da aplicação da termografia quantitativa para estudo e avaliação de danos de fissuração permite enumerar as seguintes principais conclusões:

a. Ao se empregar a termografia quantitativa no estudo de fissuras é evidente que o grau de dano avaliado pela abertura das fissuras pode ser identificado. Essa abordagem traz importantes contribuições no estudo da degradação, uma vez que não se faz somente o mapeamento das fissuras, mas é possível categorizá-las em função de sua largura.

b. Uma vez que a aplicação da termografia para estudo de patologias se baseia nas características do fluxo térmico e em como os defeitos perturbam esse fluxo, é possível associar os resultados da inspeção termográfica com a largura das fissuras em função do padrão de comportamento e dos valores do Delta T;

c. Fissuras com maior abertura apresentam valores maiores (em módulo) de Delta T, seja no aquecimento ou no arrefecimento. Isso permite usar o Delta T para classificar a severidade das fissuras em vistorias.

d. O melhor momento para detectar e avaliar as fissuras é em torno de 150 minutos de ensaio, já na fase de arrefecimento. Nessa condição se tem os maiores valores de contraste para ambas as fissuras investigadas. Em campo essa situação é particular a cada caso, principalmente considerando o grau de exposição ao sol das fachadas do edifício. Todavia a tendência é a mesma, no sentido de avaliar a fissuração no instante inicial do arrefecimento.

e. A tendência da diminuição do fluxo térmico no arrefecimento ou a estabilização do mesmo no aquecimento tendem a diminuir os valores de Delta $T$, dificultando a classificação da severidade das fissuras. 


\section{Agradecimentos}

Os autores agradecem ao Laboratório de Ensaio de Materiais e ao Laboratório de Termografia do Depto de Eng. Civil e Ambiental da Universidade de Brasília pela viabilização e custeio desta pesquisa.

\section{Referências Bibliográficas}

ASTM 1933-99 (1999). Standard Test Methods for Measuring and Compensating for Emissivity Using Infrared, West Conshohocken, Pennsylvania, United States, 1999.

ASTM E1862-97 (2010). Standard Test Methods for Measuring and Compensating for Reflected Temperature Using Infrared Imaging Radiometers, West Conshohocken, Pennsylvania, United States, 2010.

Bauer, E., Pavón,E., Pereira, C., Nascimento, M. (2016). Criteria for identification of ceramic detachments in building facades with infrared thermography, in: J.M.P.Q. Delgado (Ed.), Recent Dev. Build. Diagnosis Tech., Springer, Singapore.

Bauer, E., Castro, E.K., Silva, M.N.B. (2015). Estimate of the facades degradation with ceramic cladding: Study of Brasilia buildings, Cerâmica. 61.

Bauer, E., Freitas, V., Mustelier, N.,Barreira, E., Freitas, S.S. (2015) Infrared thermography - evaluation of the results reproducibility, Struct. Surv. 33.

Bauer, E., Milhomem, P.M., Aidar, L.A. (2018). Evaluating the damage degree of cracking in facades using infrared thermography, J. Civ. Struct. Heal. Monit. 8.

Bauer, E., Pavón, E. Oliveira, C., Pereira,C. (2016) Facades inspection with infrared thermography: cracks evaluation, J. Build. Pathol. Rehabil. 1.

Bauer, E., Pavón, E., Barreira, E., Kraus, E. (2016). Analysis of Building Facade Defects Using Infrared Thermography: Laboratory Studies, J. Build. Eng. 6.

Flores-Colen, I., Brito, J. Freitas, V. (2011). On-site performance assessment of rendering façades for predictive maintenance, Struct. Surv. 29.

Freitas, S.S., Freitas, V., Barreira, E. (2014). Detection of façade plaster detachments using infrared thermography - A nondestructive technique, Constr. Build. Mater. 70.

Maldague, X.(2001) Theory and Practice of Infrared Technology for Nondestructive Testing, Wiley, NY, USA.

Nascimento, M., Bauer, E., Souza, J.S., Zanoni, V. (2016). Estudo da degradação por ação de agentes climáticos nas fachadas de edifícios, in: REHABEND.Burgos, Spain, 2016.

Silvestre, J.D., Brito, J. (2011). J. Ceramic tiling in building façades: Inspection and pathological characterization using an expert system, Constr. Build. Mater. 25.

Souza, J.S., Silva, A., Brito, J., Bauer, E. (2018). Analysis of the influencing factors of external wall ceramic claddings' service life using regression techniques, Eng. Fail. Anal. 83.

Souza, J.S.,Bauer, E. (2018). Influência das regiões das fachadas no processo de degradação, in: V. Freitas (Ed.), Construção2018 LIVRO ATAS, Universidade do Porto, Porto, Portugal; 2018. 